\title{
Entrelacs
}

Cinéma et audiovisuel

Jodorowsky, d'un art à l'autre

\section{Hasardeuses rencontres : bifurcations et confluences du destin dans l'œuvre de Jodorowsky}

\section{Raphaëlle Hérout}

\section{(2) OpenEdition}

\section{Journals}

Édition électronique

URL : http://journals.openedition.org/entrelacs/4698

DOI : 10.4000/entrelacs.4698

ISSN : 2261-5482

Éditeur

Éditions Téraèdre

Référence électronique

Raphaëlle Hérout, « Hasardeuses rencontres : bifurcations et confluences du destin dans l'œuvre de Jodorowsky », Entrelacs [En ligne], 16 | 2019, mis en ligne le 29 octobre 2019, consulté le 05 décembre 2019. URL : http://journals.openedition.org/entrelacs/4698; DOI : 10.4000/entrelacs.4698

Ce document a été généré automatiquement le 5 décembre 2019

Tous droits réservés 


\title{
Hasardeuses rencontres : bifurcations et confluences $\mathrm{du}$ destin dans l'œuvre de Jodorowsky
}

\author{
Raphaëlle Hérout
}

\section{Introduction}

Habitée par les motifs de la traversée et de la circulation, qui fonctionnent comme autant de pérégrinations labyrinthiques au cours desquelles les personnages mettent à l'épreuve leur destinée pour mieux en reconnaître le tracé, l'œuvre protéiforme d'Alejandro Jodorowsky donne à voir et à lire une grande variété de rencontres, qui nous semblent caractériser le fait que toute rencontre peut être capitale. Ce thème peut passer pour un héritage direct de l'esthétique surréaliste. Si l'on sait que Jodorowsky a rencontré André Breton, qu'il a fréquenté la pensée surréaliste avant de s'en distancer pour créer le mouvement Panique, il nous semble que sa façon de mettre en scène des rencontres qui interrogent le rôle du hasard et de ses inflexions possibles, constitue une façon de prolonger la tradition surréaliste qui montre la puissance du désir à l'œuvre dans ce phénomène.

2 Hantés par les effets que peut produire une rencontre sur une destinée, les surréalistes ont investi ce topos littéraire pour en explorer les vertus poétiques. Qu'elle soit fortuite, dans la pure tradition de Lautréamont, ou qu'elle se produise après avoir été désirée, dans les cas de hasard objectif, la rencontre révèle essentiellement la disponibilité de toute personne à ce qui peut arriver. De cette disponibilité naît une perception du soi comme étant en prise sur un "plus à vivre »: le sujet n'est donc pas perçu comme un tout unitaire, mais comme une instance en constant devenir, toujours négociée par rapport à une altérité. C'est en ce sens que Breton et Éluard ont lancé, en 1933, l'enquête sur la « rencontre capitale » en ces termes : « Pouvez-vous dire quelle a été la rencontre capitale de votre vie?-Jusqu'à quel point cette rencontre vous a-t-elle donné, vous donne-t-elle l'impression du fortuit? du nécessaire ${ }^{1}{ }^{~}$ Cette façon de 
saisir le caractère «capital» d'une rencontre permet d'appréhender ces éléments externes qui composent l'être et vont altérer le sujet: la contingence de la rencontre est aussi une façon d'expérimenter la contingence de l'être.

3 Ce topos - tout proche d'une mystique de la rencontre - nous semble également caractéristique du fonctionnement diégétique des œuvres de Jodorowsky. Ainsi, il nous semble qu'interroger le jeu des rencontres permet de saisir la continuité narrative des personnages et des trajectoires, dans des œuvres " pétrie[s] de heurts et de collisions ${ }^{2}$ ». Nous proposons donc d'analyser ce motif de la rencontre dans les romans de la légende familiale, L'Arbre $d u$ dieu pendu ${ }^{3}$ et L'Enfant du jeudi noir ${ }^{4}$, et dans deux longs métrages caractéristiques de l'esthétique baroque et transgressive des deux premières périodes de l'œuvre de Jodorowsky5 ${ }^{5}$ El Topo (1970) et Santa Sangre (1989), dans lesquels la question du lien filial est exploitée de manière complexe. En cela, nous envisageons la tension entre filiation et rencontre comme une dialectique entre lignée et rupture, ligne droite et ligne brisée, constitutive des inquiétudes généalogiques des œuvres de Jodorowsky.

\section{La rencontre, entre principe de vie et principe de mort}

4 Les rencontres que nous allons analyser prennent place au sein d'un récit : relatées ou mises en scène, elles interrompent le cours d'une narration et le font bifurquer vers une autre direction. Une exception est cependant notable, il s'agit des arbres généalogiques qui ouvrent les deux premiers chapitres de L'Arbre du Dieu pendu : " Les racines de mon père " et "Les racines de ma mère", ainsi que les deux derniers chapitres, «La pampa promise », montrant l'arbre du remariage de Jashé Trumper, et " Jaime et Sarah ${ }^{6}$ Felicidad », arbre menant directement à Jodorowsky. Cette figuration graphique traditionnellement verticale représente sous forme de nœuds les rencontres pour visualiser les filiations ainsi réalisées, la verticalité mimant la descendance. Mais cette représentation générationnelle linéaire vient se heurter au caractère cyclique des rencontres qui ont lieu dans ce récit, et qui font dévier les lignes droites, redéployant de nouveaux embranchements. C'est le cas notamment dès le premier chapitre, lorsque Jaime et sa fratrie assistent à un spectacle de cirque au cours duquel Fanny rencontre et reconnaît d'un même mouvement le dresseur de serpents :

Fanny s'est accrochée à la jambe de l'homme noir et s'est mise à pleurer en disant «Papa! » Il l'a prise dans ses bras et il l'a bercée. Elle n'a pas tardé à s'endormir, en souriant. L'homme noir nous a dit: «Dans une vie antérieure, loin dans le temps, j'ai réellement été son père, un bon roi. Elle était un prince savant qui s'appelait Rahula ${ }^{7} . .$. [...] Maintenant, il nous faut partir. Bientôt, malade comme je le suis, je rendrai l'âme... Quand cette enfant aura dix-sept ans, elle sera ma mère. Mais moi, lassé de toutes ces réincarnations, je ne vivrai que neuf mois dans son ventre et je mourrai pendant l'accouchement... $\|^{8}$

Cette rencontre fortuite, sorte d'intermède entre deux relations passionnées, montre la façon dont ces rencontres itératives, liées à différentes incarnations, vont jusqu'à questionner la consistance des individus. Entre mémoire du passé et prégnance du futur, l'individu se compose dans ses relations et s'accomplit au fur et à mesure des rencontres. Le récit, lui, propose les boucles que l'arbre généalogique ne permet pas, pour que ces retours du passé puissent survenir sous les formes d'un nouveau présent. Le cinquième chapitre met ainsi en scène ces retrouvailles entre Fanny-Rahula et son père-fœetus : 
Fanny découvrit qu'elle pouvait méditer jambes croisées comme ses bouddhas. Dans cette position, un petit matin où elle se reposait après avoir scrupuleusement comptabilisé les bénéfices et les frais de la journée, elle entendit le message télépathique du fotus :

- Tu te souviens de moi? La dernière fois que nous nous sommes vus, c'était en Russie et tu étais une petite fille. Je me suis présenté comme un dresseur de cobras. Je t'avais dit que dans une vie antérieure, où j'avais été le roi ton père, tu t'appelais...

- Rahula! C'est vrai, je me rends compte maintenant que je ne t'ai jamais oublié.

- Notre histoire est longue. Dans des vies plus lointaines encore, toi aussi tu as été mon père, ma mère, mon frère et ma sœur, ma femme, ma maîtresse, mon maître. Nous sommes passés par presque toutes les formes de réalisation de l'amour. Nous n'avons plus rien à faire dans ce monde. Dans la prochaine transformation, nous serons une même entité ; nos âmes, enfin amalgamées, aideront à la gestation d'un univers nouveau, plus conscient que celui-ci. La seule chose que je n'ai pas encore été pour toi, c'est un enfant mort-né, présent dans ton esprit tous les jours qui te restent à vivre. Ce sera l'amour le plus fort, celui de la mère frustrée 9 .

Le fait d'avoir épuisé les formes d'amour possible va marquer la fin d'une existence, audelà des formes par lesquelles cette existence s'est matérialisée. Cette façon d'explorer l'existence à partir d'une variation d'incarnations corporelles et psychiques peut se penser comme une façon de déterritorialiser l'individu même. L'identité n'est plus liée à un corps, un espace ou un temps, mais se développe et se résorbe à travers une continuité affective ; l'existence est donc perçue dans son devenir, et non pas dans l'individu qui lui donne corps.

7 À l'inverse de l'exemple de Rahula, parfois dans le récit les personnes ne se sont pas encore rencontrées, mais leurs ancêtres ont préparé cette rencontre. C'est ce qui apparaît lors du mariage entre Jashé et Alejandro :

Lorsque ma grand-mère lui demanda pourquoi il était chaussé d'aussi scandaleuse façon [avec des « bottines d'un rouge criard»], il sut que la rencontre de leurs deux âmes, qui pouvait sembler le pur fruit du hasard, avait été préparée depuis des siècles ${ }^{10}$.

Nous retrouvons également le principe de la préparation de la rencontre dans le point de convergence des quatre arbres généalogiques représentés. Cette fois elle n'est pas liée aux ancêtres, mais à la volonté d'exister du narrateur. En effet, les arbres convergent vers la rencontre désirée des deux personnes qu'Alejandro Jodorowsky s'est choisies pour parents, et qui constitue le point d'aboutissement de la narration.

J'ai eu beaucoup de mal à faire se rencontrer Jaime et Sarah Felicidad. Lorsque le dessein de m'incarner à nouveau dans ce monde est devenu un besoin, celui que j'ai choisi comme père traînait dans un cirque où il se faisait suspendre par les cheveux $^{11}$, dans l'extrême sud du Chili, tandis que celle qui devait être ma mère était enfermée dans un sanctuaire du désert, dans l'extrême nord. Le sud et le nord en question séparés par plus de quatre mille kilomètres ${ }^{12}$.

9 Au-delà du récit de la légende familiale et de sa mise en scène, la quête du narrateur révèle donc la puissance du désir et l'événement que celui-ci est capable de produire : le choix du père et de la mère devra permettre d'objectiver l'existence - ou du moins la naissance, à partir d'un pur désir. Au niveau narratif, la concaténation des rencontres et le hasard des pérégrinations participent donc d'une logique de l'événement qui permet une circulation de l'existence. La rencontre capitale, dans ce cas-là, n'est pas celle que le narrateur a faite, mais celle qu'il a provoquée. 
10 Le récit va parfois fonctionner comme une mise à l'épreuve de ce désir et de la possibilité de son objectivation, pour laquelle le personnage va devoir trouver des ressources propres. C'est le cas par exemple lorsque, sortant de la mine du Huara, Jaime s'apprête à se suicider : pour protéger les mineurs sympathisants communistes qui ont été dénoncés, Teresa, accompagnée de ses camarades Sofia et Elias et donc aussi de Jaime, lance son véhicule à toute vitesse pour percuter celui de la milice aux ordres du dictateur, qui vient les arrêter :

La collision était imminente et le corps de mon futur père allait être déchiqueté. J’ai commencé à protester. Tous mes efforts pour l'approcher de La Tirana, où l'attendait celle que je voulais pour mère, allaient être inutiles. Merde, ce jeune homme allait droit à la mort et JE VOULAIS NAÎTRE ! Désespéré, je suis sorti de la cachette que je m'étais aménagée dans les testicules de Jaime et je suis allé chercher le Rebbé. Celui-ci a tout de suite compris la situation. Il a été horrifiéér.

11 Créer la rencontre des deux parents pour pouvoir exister nécessite donc que le principe de vie sorte victorieux d'une lutte contre le principe de mort: c'est bien le caractère éclatant du désir de vivre qui va interférer sur l'histoire en cours, en l'occurrence faire en sorte que Jaime saute du véhicule en mouvement pour sortir de la trajectoire initiée, s'arrêter, et prendre un autre chemin.

12 Parfois aussi dans le récit, la mort symbolique est une étape nécessaire qui accompagne la rencontre. C'est ce qui va sauver Fenix (Adan Jodorowsky pour Fenix enfant, puis Axel Jodorowsky pour Fenix adulte) à la fin de Santa Sangre. Après avoir vu son père (Guy Stockwell), émasculé par sa mère (Blanca Guerra), se suicider avant de s'être vengé en coupant les deux bras de sa mère, Fenix vit interné, dans un état d'aliénation totale, subissant le double joug de la folie et de sa mère. Quand il entend celle-ci l'appeler par la fenêtre de sa cellule, il répond aussitôt à son appel; commence alors une vie extérieure, en dehors de l'asile. Mais cette vie extérieure est en réalité une vie cloîtrée dans les désirs de la mère. En effet, Fenix s'unit avec elle pour pallier l'absence de ses bras, et fait de son propre corps le substitut du manque de sa mère, qu'elle manifeste de façon tyrannique.

13 Cette relation traumatique met le réel à distance dans la mesure où la mère s'arroge le pouvoir de définir le désir de Fenix et de modeler sa réalité. Complètement englouti dans cette aliénation, obéissant malgré lui aux injonctions de sa mère, Fenix tuait - au couteau ou au sabre - les femmes avec qui il se liait. Quand, après Ruby la vierge (Gloria Contreras) et Santa la catcheuse (S. Rodriguez), c'est Alma (Faviola Elenka Tapia pour Alma enfant, puis Sabrina Dennison pour Alma adulte) qu'il voit de ses yeux et qui n'offre pas de résistance aux assauts des lames, alors seulement Fenix est capable de retourner le couteau contre sa mère et lui-même, avant de se rendre compte que ce meurtre, uniquement symbolique, le libère d'un lien imaginaire: sa mère était bel et bien morte au moment de l'accident au cirque, c'est une statue de cire qui incarnait sa présence.

La relation à Alma permet ainsi de rompre l'aliénation, et donc de suspendre la logique de destruction initiée, c'est-à-dire qu'Alma ${ }^{14}$ va constituer une altérité qui va lui permettre de retrouver son identité. Santa Sangre donne donc à voir une persistance du lien à travers le temps et l'espace qui densifie l'identité des personnages. La continuité affective qui relie les jeunes Fenix et Alma, de leur enfance au cirque jusqu'à leur vie d'adulte à Mexico, agit comme un révélateur de l'identité des protagonistes et leur donne un présent qui les extrait du passé traumatique. 
15 L'intimité du lien entre le meurtre symbolique et la rencontre apparaît également dans L'Arbre du Dieu pendu, lorsque Jashé Trumper, de confession juive, attend un enfant d'Alejandro Prullansky qui est, lui, orthodoxe :

le père de Jashé la déposa enceinte sans bagages ni argent devant le théâtre municipal. La renégate avait été expulsée de la communauté et enterrée dans le cimetière, symboliquement, sous la forme d'un cercueil rempli de ses vêtements et objets personnels. Maintenant pendant une semaine les membres de sa famille se jetteraient des cendres sur la tête, s'assiéraient par terre les vêtements déchirés, boiraient du bouillon de poulet et pleureraient comme si elle était morte ${ }^{15}$.

La rencontre avec le danseur, qui va donner naissance à Sarah Felicidad, engendre donc une rupture avec la communauté d'appartenance de Jashé, qui prend la forme de la mort pour la famille et d'une nouvelle naissance pour la jeune femme. La rencontre, ici, opère un changement d'identité, pas seulement parce que Sarah va changer de nom par son mariage, mais parce qu'elle va épouser une nouvelle forme de vie en dehors de la communauté qui avait imaginé - et donc cloisonné - son futur. L'unité avec sa famille est rompue, et elle va en former une autre, en s'extrayant d'une mémoire collective pour en créer une nouvelle, reliant l'individu au collectif.

La rencontre peut donc être avant tout une rupture, par laquelle est mise à l'épreuve la continuité du moi. C'est le cas également dans $\mathrm{El} \mathrm{Topo,} \mathrm{lorsque} \mathrm{le} \mathrm{personnage} \mathrm{éponyme}$ (Alejandro Jodorowsky) rencontre la femme qu'il va sauver (Mara Lorenzio). Il met fin à son errance dans le désert avec son fils (Brontis Jodorowsky pour le fils jeune, Robert John pour le fils adulte), abandonne ce dernier à la mission franciscaine dans laquelle se déroule la scène, et poursuit son chemin avec la femme.

18 Le cowboy-père, qui accompagnait son enfant dans une quête initiatique - marquée dès le début du film par l'enterrement de son premier jouet et du portrait de sa mère devient cowboy-amant, qui va accéder à la demande de sa maîtresse de s'initier en affrontant les quatre maîtres du revolver. Avec la rencontre de la femme et la mise à mal du lien filial, les rôles changent et le personnage, qui guidait son fils, devient celui qui est guidé, jusqu'à être acteur de sa propre initiation.

19 C'est donc en étant ouvert sur l'inconnu que les personnages des œuvres de Jodorowsky font l'expérience de la constitution du moi, au travers des aléas qui surviennent sur leurs trajectoires. Les rencontres successives produisent ainsi des transformations radicales qui recomposent le moi.

20 Ces recompositions sont fécondes d'un point de vue narratif dans la mesure où elles montrent des personnages qui se détachent de ce qu'ils sont pour s'engager dans un autre devenir. Les héros de Jodorowsky présentent donc des identités mouvantes, révélant des interstices dans la subjectivité, que l'auteur ou réalisateur peut exploiter pour créer un trouble chez le lecteur ou spectateur.

21 C'est ce qui se produit lorsque El Topo part, à la demande de sa maîtresse, à la recherche des quatre maîtres du revolver, chacun installé dans un lieu inconnu du désert. Il s'agira alors pour le pistolero d'arpenter méthodiquement le désert-la méthode prend la forme symbolique de la spirale -, de trouver ces maittres, puis de les tuer. À nouveau, la rencontre et la mort sont intimement liées, dans une sorte d'équilibre par lequel le savoir une fois dévoilé rend l'existence du maitre non nécessaire. En sortant victorieux des quatre épreuves, El Topo a opéré sa transformation : se modifier soi-même en s'appropriant les savoirs d'autrui, montrant ainsi des personnages dépositaires de savoirs non pas hérités mais conquis, parfois 
brutalement. En cela, El Topo est allé dans un même mouvement à la rencontre de sa propre transformation, et à l'encontre de son instinct: tuer le premier maître a constitué une épreuve morale, mais celui-ci lui a aussi permis d'apprendre à mourir. Un cycle du film s'achève alors, le héros est prêt à se donner la mort avant d'être arrêté par sa maîtresse et son double (Paula Romo), puis d'être à son tour abandonné, laissé pour mort.

Dans cet état d'abandon, où il cesse d'exister pour lui, il va commencer à exister pour les autres : un peuple de nains et d'estropiés vivant reclus dans une grotte le prennent pour idole ${ }^{16}$ et le vénèrent. Il réapparaît donc après une période de latence sous une forme beaucoup plus proche de celle des maîtres du désert qu'il vient de tuer que de son allure de pistolero. De son existence précédente, rien ne subsiste apparemment ; la rencontre avec ce peuple de l'ombre, laissé pour compte par une société elle-même dégénérée, vient briser la linéarité temporelle jusque-là préservée. Son identité change, la seule continuité avec son état précédent sera assurée par son fils, qu'il retrouve sur son chemin dans un nouveau renversement générationnel : c'est sous les allures de moine franciscain que le fils d'El Topo réapparaît, mais dès leur mise en contact, le fils devient à son tour pistolero et devra se charger de tuer son père. La dualité entre pulsion de vie (représentée par le mariage d'El Topo, la grossesse de sa compagne (Jacqueline Luis) et la sortie du peuple hors de la grotte) et pulsion de mort (le parricide) s'écroule lorsque le fils avoue l'impossibilité de passer à l'acte en prononçant la dernière phrase du film : «How can I kill my master ? $^{17}$ ». Puisque c'est par l'esprit que le parricide est rendu impossible, c'est par le corps que le père lui-même palliera la "faiblesse » du fils, en s'immolant par le feu. Dans l'esthétique des enchaînements et renversements développée par Jodorowsky, ce point d'aboutissement ne saurait en être un, puisque la mort du protagoniste coïncide avec la naissance de son nouvel enfant, que le premier fils, devenu à son tour El Topo, emporte avec lui et la femme, vers une nouvelle errance.

Un équilibre quasi cosmique s'instaure donc au gré des rencontres, dans une sorte de balance universelle qui réagit aux événements. Le procédé est semblable dans Santa Sangre lorsque Fenix répond favorablement aux avances de Ruby la vierge. Il troque alors son costume de magicien - le même que lorsqu'il était enfant - pour la tenue rutilante de son père. Sa façon de manifester son désir à l'égard de la femme sera de devenir à son tour lanceur de couteaux, et de reproduire la scène où la femme tatouée est la cible érotique des lancers de couteaux du père. La superposition des deux scènes se fait d'abord par l'accoutrement, puis par les gestes, avant que la musique caractéristique du cirque ne vienne remplir l'espace sonore et que l'image de la scène originelle, lors de laquelle Fenix-enfant est spectateur, ne vienne temporairement prendre le relai de la scène vécue, dans laquelle Fenix-adulte est acteur.

Cette résurgence du père à travers le fils participe donc également de la dialectique autonomie-aliénation : le fils supplante le père et en devient un double en même temps. Fenix ou le fils d'El Topo, par leur existence, donnent une nouvelle consistance à l'image de leur père.

À travers les pérégrinations et les trajectoires souvent chaotiques, la rencontre apparaît être le révélateur de la construction de l'identité comme principe émanant de relations. C'est-à-dire que l'être se compose et se recompose en permanence, dans un mouvement général où les rencontres assurent une continuité ontologique à travers les différentes objectivations de l'existence. 


\section{La rencontre comme arrimage individuel à une histoire collective}

Entre les différentes strates de sédimentations qui se superposent à mesure qu'évoluent les personnages et les traces de l'éclatement de l'unité du moi, un autre phénomène ressort de l'enchaînement des rencontres dans les œuvres de Jodorowsky: les interférences entre l'histoire individuelle et l'histoire collective dans laquelle s'impliquent les individus. Les récits, en effet, ont tendance à imbriquer les différents niveaux d'histoire, comme en témoigne ne serait-ce que le titre L'Enfant du jeudi noir, qui est censément basé sur des faits autobiographiques. À la lecture du récit, la superposition de la naissance de l'enfant et de la catastrophe économique qui met en crise l'Occident capitaliste apparaît de manière limpide. Mais comme le remarque Caroline Lepage, "on peut s'étonner [...] que l'auteur fasse naître le personnage de Alejandro Jodorowsky le fameux Jeudi noir de 1929 [24 octobre], alors que lui-même est né le 17 février de cette même année ${ }^{18}{ }^{\prime}$. Troquer sa véritable date de naissance contre une date politiquement marquante permet d'imbriquer les deux événements et de suggérer que sa naissance s'inscrit dans un ordre mondial, comme s'il était né dans les décombres d'un monde qui a abandonné toute spiritualité, pour parer aux désordres politiques. En outre, cette superposition nous semble révélatrice de la circulation des personnages, entre légendes spirituelles, histoire de la migration et réalité prosaïque. Il s'agit donc de se situer au cœur d'un cataclysme, au moment où tout s'écroule (et est à reconstruire), où les catégories établies s'effondrent dans un renversement carnavalesque. À propos de ces interstices du mythe, de la fiction et des éléments biographiques, Caroline Lepage explique que «le mythe non seulement éclaire mais modifie le passé19 ${ }^{19}$. En termes narratifs, la confusion des strates participe de la création d'un personnage composite qui se façonne dans le désordre auquel sont soumis l'espace et le temps. Ce fantasme de l'inscription de l'histoire individuelle dans l'histoire politique est aussi une façon de noyer l'individu dans une mémoire collective, et de montrer que les événements du monde débordent de et dans l'individu.

Rattacher la légende familiale à l'histoire politique permet donc d'amarrer un récit imaginaire et collectif à des événements historiques jusqu'à ce que les deux éléments ne fassent plus qu'un. L'indécidabilité qui règne alors dans le récit s'apparente à une inquiétante étrangeté : les événements historiques familiers, désastreux et destructeurs (la fuite du continent européen pour les Juifs, la dictature argentine, entre autres), sont perçus avec un crible légendaire qui est aussi une façon de nourrir l'imaginaire.

Aussi les rencontres vont-elles être une façon d'interroger cette histoire collective en entrant dans la culture. C'est ce qu'explique Caroline Lepage :

l'omniprésence thématique et le systématisme référentiel qui en découle servent moins une réflexion d'ordre spirituel ou même philosophique que culturel, c'est-àdire [...] qui renvoie au sentiment d'appartenance à un groupe qu'en l'occurrence l'auteur s'attache à mettre en évidence, s'agissant des Juif ${ }^{20}$.

Ce sentiment d'appartenance est ce qui est mis à l'épreuve tout au long du récit, à partir de Teresa, qui fournit l'épigraphe de L'Arbre du Dieu pendu («Oh Dieu, après ce que tu nous as fait tu mérites d'être pendu $\left.{ }^{21} »\right)$ et ouvre le récit sur une rupture inaugurale : 
En 1903, Teresa, ma grand-mère, la mère de mon père, se fâcha contre Dieu et contre tous les juifs de Dniepropetrovsk en Ukraine, parce qu'en dépit de la crue meurtrière du Dniepr, ils continuaient à croire en Lui. Joseph, son fils adoré, périt dans l'inondation. Lorsque l'eau commença d'envahir la maison, le jeune garçon poussa un bahut dans la cour pour grimper dessus, mais le meuble, où s'entassaient les trente-sept volumes du Talmud, refusa de flotter...22

30 jusqu'à vivre comme un saint, accomplissant des miracles : «Là, sur ce pas de porte misérable, il resta deux ans à guérir sans interruption tout malade qui le lui demandait. Le Rebbé n'avait rien à voir avec ces miracles. Sa monture avait atteint la saintetée ${ }^{3}$. » présentes jusqu'à un certain enfermement qui le détachera de l'existence, alors que Teresa va toujours se projeter dans un ailleurs, se précipiter vers toutes les issues possibles, pour se réinstaller dans une existence qui n'a plus rien à voir avec celle de sa vie sur le continent européen. Dès lors, Alejandro fera une succession de rencontres spirituelles qui le guideront dans sa quête mystique (parcourir quatre mille kilomètres à pied pour se rendre à Buenos Aires afin de ramener une Thora dans sa synagogue), tandis que Teresa rencontrera la personne qui l'épanouira dans sa vie charnelle. La rupture avec Alejandro et la culture juive est donc ce qui permet un renouveau à Teresa : «Ne me cherche pas, parce que la Teresa que tu as connue n'existe plus. Dis-toi que je suis morte. Les enfants n'ont plus besoin de moi $^{24}$.» Sortir de sa première vie est également synonyme, pour Teresa, de se doter d'une mémoire individuelle, ce qui, pour Caroline Lepage, est synonyme de recherche sur l'identité culturelle :

En outre, pour définir son identité culturelle, Jodorowsky réfléchit sur la tentation de tourner le dos à Dieu et à la communauté hébraïque, en somme à ses croyances mais aussi à ses origines, plus essentiellement encore à sa propre mémoire... dès lors que cette mémoire individuelle est montrée comme intrinsèquement liée à la mémoire collective ${ }^{25}$.

Dès lors, les différentes rencontres mèneront les personnages à intégrer d'autres mémoires, et à leur faire jouer un rôle sur le présent, comme lorsque Jaime rencontre Tralaf, un Indien Mapuche qui va le soigner, justement en l'intégrant à sa propre mémoire : «Cela fait beaucoup de siècles que tu as perdu le paysage et sans racines, pas de santé26." Tralaf va donc partager ses racines mapuches avec Jaime lors d'une initiation physique et spirituelle, avant de le faire participer à sa cosmogonie: «Courage, huinca. Notre aigle du soleil est venu. Tu n'es pas seul. Il y a en toi l'âme d'un ancêtre. Il te soutient ${ }^{27}$."

Il en ira de même lorsque Jaime rencontrera Recabarren ${ }^{28}$ et, avec lui, la culture marxiste. C'est d'abord grâce à son nom et, par là, ses origines, que le lien se crée :

- Comment vous appelez-vous?

- Jaime Jodorowsky, à votre service.

- Nom bizarre. Polonais?

- Oui, mais ma famille est russe... Je mens... Elle est juive.

- Mais, vous savez parler russe?

- J'ai la chance de ne pas l'avoir oublié.

- Voulez-vous travailler avec moi ?

- Bien sûr !29

L'origine n'est plus liée à la religion mais à la langue et à la politique et, grâce à ces origines, Jaime va pouvoir envisager une nouvelle vie : « quelque chose lui disait qu'en franchissant ce seuil, sa vie allait changer. Il avait besoin de trouver des racines qui le 
tirent de la folie, de jeter l'ancre dans un fond solide ${ }^{30}$ ». Et ce fond prendra la forme d'une famille :

- Je suis Jaime Jodorowsky. Monsieur Recabarren m'a proposé un travail.

- Ah, le jeune homme qui parle russe ! [...] Entrez. Je suis Teresa, sa compagne.

Teresa! Cette femme s'appelait comme sa mère. Il venait d'en perdre une et la magie du hasard lui en fournissait une autre, peut-être meilleure ${ }^{31}$.

Une fois cette reconstitution familiale accomplie, une intégration totale commence, lui donnant accès à une identité de substitution.

- Il nous faudra vous trouver un nom. À cause des persécutions, chaque camarade doit s'inscrire sous un pseudonyme. Un nom mapuche, Lautaro Quinchahual par exemple, ça vous dirait?

Cette fois le pays lui ouvrait une porte pour de bon [...]. On le baptisait une deuxième fois, on le faisait naître dans une patrie, on l'acceptait comme aborigène, on lui donnait un peuple frère ${ }^{32}$.

Une fois intégré à ce "peuple frère " qui le met en contact avec l'histoire sociale du Chili et de sa soumission à l'économie nord-américaine, c'est la volonté de naître de son fils qui l'arrachera à sa famille d'adoption pour constituer sa propre famille. Dès lors, il ne s'agira plus seulement d'entrer dans l'histoire collective du pays, mais aussi de tenter d'intervenir sur les événements historiques.

L'individu reprend le pas sur le collectif et veut le marquer de son empreinte. Cela correspond au moment où Jaime laisse Sara Felicidad et les deux enfants pour accomplir ce qu'il considère comme sa mission : tuer le dictateur Ibañez, pour libérer le pays de son pouvoir autoritaire et des violences arbitraires qu'il engendre. C'est alors que l'idée de contingence redisparaît, laissant place à la nécessité de son action :

Jaime n'avait pas de plan précis mais, ayant vu ce qu'il avait vu, étant passé par où il était passé, il ne niait plus les possibilités miraculeuses de la réalité. Il se rendait compte que tout était lié par des fils invisibles, comme un tissu dépendant d'une conscience infinie, toute-puissante et prompte à obéir... Pour peu qu'on ait l'audace de s'en proclamer le maître et de la mettre fermement en ordre, en réunissant tous les moyens pour la réalisation de ce dessein, et sans qu'importent ni le bien ni le mal. Ce qui comptait dans ce plan, ce n'était pas la qualité morale du héros mais la force de sa volonté. Et cette force-là, il l'avait, il en était certain, en tout cas autant que ce maudit dompteur de chevaux. Ce qui allait faire pencher le plateau de la balance en sa faveur, en dehors de l'astuce, c'était le miracle ${ }^{33}$.

Mais l'idée de maîtrise et de volonté apparaissent bien vite comme des illusions :

Il semblait que la trame invisible soutenant le présent qui lui était donné de vivre se resserrait jusqu'à ne plus devenir qu'un fil unique, ou plus exactement une solide corde, dans le but de s'enrouler autour de lui et de le trainer, sans détails superflus, vers l'œil du cyclone de son destin ${ }^{34}$.

"L'œil du cyclone » apparait alors comme un moment de pleine appartenance au monde et à soi-même. C'est également le moment de cristallisation de tous les possibles où le temps s'intensifie, sort du chronos pour apercevoir le kaïros. Mais Jaime restera au bord du kaïros sans le saisir et sans se saisir d'une vengeance expéditive, et il ne fera pas basculer l'histoire à ce moment-là, prisonnier qu'il est des fils qui dirigent son destin :

Comme un pion mû par des dieux sur un échiquier cosmique, Jaime avait rapidement atteint ses deux principaux objectifs: gagner la confiance d'Ibañez et devenir le palefrenier de son cheval préféré, c'est-à-dire du seul être devant lequel le tyran abandonnait sa froide cuirasse et montrait un cœur vulnérable... Jaime ne tirait cependant pas fierté d'un tel succès parce qu'en réalité, il ne le comprenait pas ${ }^{35}$. 
41 Les conditions étaient réunies pour que Jaime tue Ibañez, mais il ne l'a pas fait. Ayant échoué, c'est son être entier qui se dissout: "Immobile, Jaime resta au milieu du chemin, dans un brouillard de terre [...]. Lorsqu'on n'entendit plus le moteur et que le tourbillon de poussière se dissipa, il n'était plus Jaime. Il avait perdu la mémoire ${ }^{36}$. » Dès lors, le chemin qui le ramènera, bon gré mal gré, à Sara Felicidad, s'apparente à la fois à une descente aux enfers et au retour d'Ulysse à Ithaque, deux épreuves qui permettront aux trois membres de la famille de se reconnaître et de trouver, finalement, leur identité. Il s'agit de retrouver son nom: doña Sara redevient Sara Felicidad, Jaime parviendra à nommer son fils par son véritable prénom, Alejandro, et celui-ci à appeler son père «papa »; le nom, en ce sens, permet la singularité.

À l'inverse de cet assassinat manqué, El Topo montre une libération ratée, qui se conclut elle aussi sur une dissolution des sujets individuels et collectifs : lorsque le protagoniste parvient à ouvrir la paroi séparant la grotte du monde extérieur, le sentiment de liberté retrouvée pousse la femme à l'intérieur de la grotte pour prévenir le reste des habitants, contre l'avis d'El Topo : "You can't tell them, they're not ready for it ${ }^{37}$." L'enjeu est ambivalent, puisqu'El Topo sait que le moment de la libération des habitants coïncidera avec celui de son exécution par son fils. Le fait de retarder l'annonce de l'ouverture de la grotte pourrait passer pour un moyen de retarder sa mort, mais en réalité c'est bien de celle du peuple «freak » qu'il s'agit, car une fois la grotte ouverte, le peuple se précipite vers le village à la rencontre d'un espace occupé par une bourgeoisie dégénérée - celle-là même qui avait enfermé les estropiés. Cette rencontre entre les bourgeois, qui détiennent à la fois le pouvoir symbolique (celui de bannir de la ville et de faire disparaître de leurs perceptions une partie de la population) et le pouvoir des armes (qui fait qu'ils décident du droit de vie ou de mort), et le peuple « freak ", au cours de laquelle les habitants de la grotte vont être massacrés, montre donc une émancipation impossible. La violence de la scène finale de fusillade, qui trouve son point d'aboutissement dans l'immolation d'El Topo, fait écho aux exécutions sommaires $\mathrm{du}$ début $\mathrm{du}$ film, qui apparaissent comme un motif politique face auquel les personnages vont construire leur trajectoire.

\section{Conclusion}

Le jeu des rencontres, en interrogeant les identités et en maintenant la tension entre contingence et nécessité, participe donc d'une esthétique syncrétique qui multiplie les voies d'emprunt possibles pour les personnages et décuple les effets symboliques. Dans ces errances initiatiques, révélatrices des boucles du destin, le phénomène de la rencontre permet de mettre à l'épreuve la présence du personnage, sa participation à son présent. La dialectique de l'altération et de la révélation du moi semble donc un élément structurant dans les récits et films de Jodorowsky, elle provoque une mise en tension qui anime les personnages. Lorsque Jaime prend la peine d'expliquer à Gamboa le boiteux : "comprenez [...] que l'individu est une illusion ${ }^{38}$ ", on comprend que les fictions fonctionnent comme une plongée au cœur de cette illusion, en faisant varier les différents systèmes symboliques permettant de l'appréhender.

Le caractère labile de l'individu fait que, s'il reste repérable dans l'espace, il perd son ancrage dans le temps. La figure même de l'arbre généalogique garde sa force d'évocation mais change de forme sous l'influence de représentations cycliques : «Sara Luz lui expliquait que le passé était une invention perpétuelle, que chaque personnage 
sur l'arbre pouvait être comparé à une pierre qui, au fil des ans et de récit en récit, s'élevait jusqu'au ciel et brillait comme un astre ${ }^{39}$ ». Le récit interfère sur la perception du réel, créant les conditions pour engager les personnages vers des séries de recommencements propices à une nouvelle perception légendaire du passé. Les rencontres concourent donc à brouiller toute notion de linéarité du temps et d'unité du sujet: les personnages sont entièrement en relation non seulement avec le passé et l'avenir, mais aussi avec d'autres formes possibles de leur existence.

\section{NOTES}

1. André Breton et Paul Éluard, «Enquête », dans Minotaure, n³-4, 1933, p. 101-115.

2. Charlotte Serrus, «La bibliothèque de midi », La Pensée de midi, vol. 30, n 1, 2010,

p. $135-162$.

3. Alejandro Jodorowsky, L'Arbre du dieu pendu, Paris, Métailié, 1996.

4. Alejandro Jodorowsky, L'Enfant du jeudi noir, Paris, Métailié, 2000.

5. La première correspond au tournant des années 1970, avec notamment Fando et Lis (Fando y Lis, 1968), El Topo, et La Montagne sacrée (La Montaña sagrada, 1973), puis, après l'abandon de Dune (1973-1977, pour le projet), la deuxième période se situe au tournant des années 1990, avec Santa Sangre et Le Voleur d'arc-en-ciel (1990), puis la dernière période commence dans les années 2010, avec La Danza de la realidad (2013) et Poesía sin fin (2016).

6. La Sarah Felicidad de L'Arbre du dieu pendu devient Sara Felicidad dans L'Enfant du jeudi noir.

7. Cette mention, qui convoque la culture bouddhiste, participe du syncrétisme propre à Jodorowsky.

8. Alejandro Jodorowsky, L'Arbre du dieu pendu, op. cit., p. 47-48.

9. Ibid., p. 338-339.

10. Ibid., p. 61.

11. Ce que fait la mère de Fenix dans Santa Sangre.

12. Ibid., p. 305.

13. Ibid., p. 460.

14. «Alma » signifie « âme » en français.

15. Ibid., p. 61.

16. Il en va de même pour Jaime dans L'Enfant du jeudi noir, qui est retrouvé dans le coma par « la pendue », qui veillera sur lui pendant sept ans.

17. En version originale, sous-titré par : «Je ne peux pas tuer mon maître. »

18. Caroline Lepage, « Légende familiale et identité culturelle dans Donde mejor conta un pájaro et El niño del Jueves negro de Alejandro Jodorowsky ", dans America, vol. 30, $\mathrm{n}^{\circ} 1$, 2003, p. 91-97.

19. Ibid.

20. Ibid., p. 92.

21. Alejandro Jodorowsky, L'Arbre du dieu pendu, op. cit., p. 7

22. Ibid., p. 13. 
23. Ibid., p. 190.

24. Ibid., p. 168.

25. Caroline Lepage, « Légende familiale et identité culturelle dans Donde mejor conta un pájaro et El niño del Jueves negro, d'Alejandro Jodorowsky », op. cit., p. 92.

26. Alejandro Jodorowsky, L'Arbre du dieu pendu, op. cit., p. 381.

27. Ibid., p. 382.

28. Jaime errait de village en village en portant une croix, suscitant ainsi la compassion et la générosité des gens qu'il rencontrait, jusqu'à ce qu'une chute (et « un sacré coup de christ sur sa clavicule, qui se déboîta ») interrompe son chemin. Il est alors recueilli et soigné par un fossoyeur, Don Eleodoro, qui lui offre le costume d'un homme récemment mort. Jaime poursuit sa route dans son costume, jusqu'à se rendre au « Premier Congrès du Parti Communiste du Chili », où il sera reconnu par Recabarren car le costume qu'il porte est en fait celui, reconnaissable par sa couleur vive, de son oncle. Par ce costume, Jaime et Recabarren sont déjà liés : "Comme vous voyez, grâce à vos habits voyants, nous sommes presque de la même famille ». (Ibid., p. 419)

29. Ibid.

30. Ibid., p. 425.

31. Ibid., p. 426.

32. Ibid., p. 429.

33. Alejandro Jodorowsky, L'Enfant du jeudi noir, op. cit., p. 107-108.

34. Ibid., p. 109.

35. Ibid., p. 113.

36. Ibid., p. 124.

37. « Ne leur dis pas encore. Ils ne sont pas prêts ».

38. Ibid., p. 93.

39. Ibid., p. 73.

\section{RÉSUMÉS}

Nous proposons une lecture croisée des deux romans de la légende familiale (L'Arbre du Dieu pendu et L'Enfant du jeudi noir) ainsi que des deux films antérieurs aux années 2000 dans lesquels la question du lien filial est centrale (El Topo et Santa Sangre), à partir de la question de la rencontre. Plus précisément, nous envisageons la rencontre comme un événement qui, dans la tradition surréaliste, interroge les notions de hasard et de nécessité et révèle les paradoxes d'un type de personnage qui est le fruit agi de ces rencontres et qui s'affirme au fur et à mesure de ces rencontres. La question sous-jacente est celle de la constitution de l'identité du sujet: personnage ouvert sur l'inconnu, dont les désirs œuvrent à la découverte de cet inconnu, le protagoniste des œuvres d'Alejandro Jodorowsky incarne, par sa disponibilité à la rencontre, une expérience forte de constitution du sujet et de continuité du moi par et au travers des aléas qui informent sa trajectoire.

We propose a cross-reading of the two novels of the family legend (L'Arbre du Dieu perdu and L'Enfant du jeudi noir), as well as the two films prior to the 2000s in which the question of the filial link is central (El Topo and Santa Sangre), starting from the question of the encounter. More 
precisely, we envisage the encounter as an event that, in the surrealist tradition, questions the notions of chance and necessity and reveals the paradoxes of a type of character who is the fruit of these encounters, and who asserts himself over the course of these encounters. The underlying question is the constitution of the identity of the subject: character open to the unknown, whose desires work to discover this unknown, the protagonist of Jodorowsky's works embodies, by his availability to encounter, a strong experience of the constitution of the subject and of the continuity of the self by and through the hazards that inform his trajectory.

\section{INDEX}

Mots-clés : Rencontre, hasard, trajectoire, identité, filiation, narration

Keywords : Rencontre, hasard, trajectoire, identité, filiation, narration

\section{AUTEUR}

\section{RAPHAËLLE HÉROUT}

Ater à l'université de Caen, Raphaëlle Hérout est docteure en langue et littérature française et a consacré sa thèse à l'imaginaire linguistique du surréalisme. Elle travaille sur les avant-gardes, l'énonciation poétique et les rapports entre normes langagières et normes sociales. 